\title{
Global Chaotic Parameters of Heart Rate Variability During Mental Task
}

\author{
ANNE M. G. FONTES, ${ }^{1}$ DAVID M. GARNER, ${ }^{2}$ LUIZ CARLOS DE ABREU, ${ }^{3}$ JULIANA C. BARBOSA, ${ }^{1}$ \\ ELISANGELA VILAR DE ASSIS, ${ }^{3}$ ANA CECÍLIA A. DE SOUZA, ${ }^{3}$ \\ ANDREY A. PORTO, ${ }^{1}$ AND VITOR E. VALENTI ${ }^{1}$ \\ ${ }^{1}$ Faculdade de Filosofia e Ciências, Departamento de Fonoaudiologia, Centro de Estudos do Sistema \\ Nervoso Autônomo (CESNA), UNESP. Av. Hygino Muzzi Filho, 737. 17525-000 Marília, SP, Brazil; \\ ${ }^{2}$ Faculty of Health and Life Sciences, Department of Biological and Medical Sciences, Cardiorespiratory \\ Research Group, Oxford Brookes University, Gipsy Lane, Oxford OX3 OBP, United Kingdom; and ${ }^{3}$ Facul- \\ dade de Medicina do ABC. Departamento de Morfologia e Fisiologia, Av. Príncipe de Gales, 821. \\ 09060-650 Santo André, SP, Brazil
}

Received 28 July 2014; accepted 8 January 2015

\begin{abstract}
We aimed to evaluate the novel chaotic global techniques of heart rate variability (HRV) analysis during a specific autonomic test, the mental arithmetic overload test. These are spectral detrended fluctuation analysis and spectral multi-taper method; in addition to spectral entropy. We analyzed 24 healthy male students-all nonsmokers, aged between 18 and 22 years old. HRV was analyzed in the following periods: control protocol-the 10-min periods before the performance of the task and the 5-min periods during the performance of the test. Following tests for normality; Kruskal-Wallis technique and principal component analysis-it was decided that this type of mental stimulation did not lead to significant changes in any of the seven combinations of chaotic globals. In conclusion, it was suggested that the time-series be increased to 1000 RR intervals (at least 20 min of electrocardiographic data) and standard nonlinear methods be introduced in combination with spectral factors as a way of increasing the statistical significance. () 2015 Wiley Periodicals, Inc. Complexity 21: 300-307, 2016
\end{abstract}

Key Words: cardiovascular system; autonomic nervous system; mental stimulation; chaos

Additional supporting information may be found at the online version of this article

Correspondence to: Vitor E. Valenti, Departamento de Fonoaudiologia, Faculdade de Filosofia e Ciências, UNESP. Av. Hygino Muzzi Filho, 737. 17525-000, Marília, SP, Brazil. E-mail: vitor.valenti@marilia.unesp.br

\section{INTRODUCTION}

4 he RR intervals of the electrocardiographic (ECG) PQRST waveform can fluctuate in an apparently chaotic manner [1]. Methods derived from statistical mechanics have motivated research in this area [2]. Heart rate variability (HRV) analysis using standard temporal separations of the time-series or indeed the new chaotic 
globals techniques [3] is an important emerging field of study. The assessment of these methods has coined the term "dynamical disease study [4]." Conventional techniques datasets usually take from days to weeks [5]. Here, we are applying chaotic global techniques over 256 RR intervals or approximately 4-6 min.

Detrended fluctuation analysis (DFA) [6-8] and Shannon entropy [9-11] are the traditional algorithms to be applied to time-series. Adaptations of these techniques include spectral detrended fluctuation analysis (sDFA) [3] and spectral entropy [12]. Additionally, we assess the spectral multi-taper method (sMTM) [3,13]; which is based on the variation of the power spectrum due to broadband noise elevation during a chaotic response.

These techniques have been useful to clinicians assessing conditions when the patient is uncommunicative such as sleep apnea [14] and surgical patients under anaesthesia [15]. In this sense, cardiac autonomic regulation may also be tested through autonomic tests such as and mental tasks [16]. These tests are important means of diagnosing autonomic dysfunction [16,17].

Here, we consider subjects undergoing mental stimulation by undertaking mathematical problems. Similar studies have been assessed in subjects with diabetes mellitus [13] and childhood obesity [18]. Therefore, this study aimed to investigate the acute effects of a specific mental task on the globally chaotic parameters of HRV.

\section{METHOD}

\subsection{Study Population}

The subjects were 24 healthy male students-all nonsmokers, aged between 18 and 22 years old. All volunteers were informed about the procedures and the objectives of the study and gave written informed consent. All study procedures were approved by the Ethics Committee in Research of the Faculty of Sciences of the Universidade Estadual Paulista, Campus of Marilia (No. CEP-2011-385) and were in accordance with Resolution 196/96 National Health 10/10/1996.

Exclusion criteria included body mass index (BMI) $>35 \mathrm{~kg} / \mathrm{m}^{2}$; systolic blood pressure $>140 \mathrm{mmHg}$ or diastolic blood pressure $>90 \mathrm{mmHg}$ (at rest); cardiac arrhythmias (atrial flutter or fibrillation, multiple ventricular or atrial ectopy, second or third degree atrioventricular block); smoking, left ventricular dysfunction; reported neurological, or respiratory disorders; and, relevant auditory disorders.

\subsection{Initial Evaluation}

Baseline information collected included: age, gender, weight, height, and BMI. Weight was determined using a digital scale (W 200/5, Welmy, Brazil) with a precision of $0.1 \mathrm{~kg}$. Height was determined using a stadiometer (ES 2020, Sanny, Brazil) with a precision of $0.1 \mathrm{~cm}$ and $2.20 \mathrm{~m}$ of extension. BMI was calculated as weight $/$ height $^{2}$, with weight in kilograms and height in meters.

\subsection{The Mental Arithmetic Overload Test}

The task consisted of continuous mental operations with two or three digits (i.e., $287+24-43 / 3+28$ ) for 5 $\min$. The test had to be performed without verbal stimulation. (Supporting Information).

\subsection{HRV Analysis}

The RR intervals recorded by the portable HR monitor (with a sampling rate of $1000 \mathrm{~Hz}$ ) were downloaded to the Polar Precision Performance program (v.3.0, Polar Electro, Finland). The software enabled the visualization of $\mathrm{HR}$ and the extraction of a cardiac period (RR interval) file in "txt" format. Following digital filtering complemented with manual filtering for the elimination of premature ectopic beats and artefacts, $256 \mathrm{RR}$ intervals were used for the data analysis. Only series with sinus rhythm greater than $95 \%$ were included in the study.

HRV was analyzed during four time periods: the 10min period before the test without mental stimulation, the 10 -min period before the test with mental stimulation, the 5 -min period during the test, and the 5 -min period after the test. The geometric indices of HRV were evaluated. For calculation of the indices, we used HRV Analysis software (Kubios HRV v.1.1 for Windows, Biomedical Signal Analysis Group, Department of Applied Physics, University of Kuopio, Finland).

\subsection{Protocol}

Data collection was undertaken in the same soundproof room for all volunteers; the temperature was between $21^{\circ} \mathrm{C}$ and $25^{\circ} \mathrm{C}$ and the relative humidity was between $50 \%$ and $60 \%$. Volunteers were instructed not to drink alcohol, caffeine, or other autonomic nervous system (ANS) stimulants for $24 \mathrm{~h}$ before the evaluation. Data were collected on an individual basis, always between 18:00 and 21:00 to avoid circadian influences. All procedures necessary for the data collection were explained to each subject separately, and the subjects were instructed to remain at rest and avoid talking during the collection.

HRV was analyzed in the following periods: control protocol-the 10-min periods before the performance of the task and the 5-min periods during the performance of the test.

\subsection{Chaotic Assessment}

Since the time-series are extremely short, we must apply power spectra to the data. Applying such algorithms converge faster than computed on interpeak temporal separations. Precision is increased for any fine detailed structure when we use Welch method [19] for spectral entropy or sDFA. The sMTM applies the multi-taper spectrum $[20,21]$. 


\subsection{Spectral Entropy}

Spectral entropy $[22,23]$ is a function of Shannon entropy. It provides us with a value that characterizes the probability that different power spectral outputs of length $N$ occur, where, $p_{i}$ is the probability of being in state $i$. Spectral disorder is a function of the irregularity of amplitude and frequency of the peaks in power spectra.

$$
S_{0}=-\sum_{i=1}^{N}\left(p_{i}\right) \log _{2}\left(p_{i}\right) .
$$

We calculate the power spectrum by Welch's method [19] setting the Welch's method parameters at: sampling frequency of $1 \mathrm{~Hz}$; zero overlap; a Hamming window with FFT length of 256 and, no detrending. This output is then manipulated so that the sum of the magnitude is equal to unity; giving a normalized power spectrum. From here, we evaluate Shannon entropy of the frequencies from normalized power spectra. We then calculate an intermediate parameter which is the median Shannon entropy of the three different power spectra using the Welch power spectra under three test conditions: (i) a perfect sine wave, (ii) uniformly distributed random variables, and finally, (iii) the experimental oscillating signal. These values are then normalized mathematically so that the sine wave gives a value of zero, uniformly distributed random variables give unity, and the experimental signal between zero and unity. It is this final value that corresponds to spectral entropy.

\subsection{SDFA}

DFA $[7,24]$ can be applied to datasets where statistics such as mean, variance, and autocorrelation vary with time. The difference with the sDFA [3] algorithm is that the DFA is applied to the frequency rather than time on the horizontal axis. Regarding DFA according to Donaldson [25] the time-series of length $k$ was integrated as follows.

$$
y(k)=\sum_{i=1}^{k}(x(i)-\bar{x}) .
$$

The integrated time-series was then divided into equally sized and nonoverlapping windows of length $w$. A linear regression line was fitted through the data in each window and the time-series locally detrended by subtracting the regression line from the data. The root mean square fluctuation $F(w)$ of the integrated and detrended time-series was then used to calculate values of $w$.

$$
F(w)=\left[\frac{1}{N} \sum_{k=1}^{N}\left[y(k)-y_{w}(k)\right]^{2}\right]^{\frac{1}{2}}
$$

The scaling exponent obtained as the slope of the straight line fit to $F(w)$ against $w$ on a log-log plot as:

\section{TABLE 1}

Mean and standard deviation for time-domain indices before (Rest), during the test (Test) and after the mental arithmetic overload test (Post test) in the control protocol

\begin{tabular}{lccc}
\hline Index & Rest & Test & $P$ \\
\hline SDNN & $42 \pm 11$ & $32 \pm 2$ & 0.52 \\
RMSSD & $22 \pm 2$ & $24 \pm 13$ & 0.89 \\
pNN50 & $7 \pm 3$ & $10 \pm 8$ & 0.64 \\
LF (ms2) & $946 \pm 657$ & $511 \pm 432$ & 0.21 \\
LF (nu) & $63 \pm 9$ & $52 \pm 11$ & 0.43 \\
HF (ms2) & $584 \pm 533$ & $345 \pm 215$ & 0.34 \\
HF (nu) & $39 \pm 14$ & $45 \pm 12$ & 0.43 \\
LF/HF & $622 \pm 122$ & $211 \pm 152$ & 0.5
\end{tabular}

SDNN = standard deviation of normal-to-normal $\mathrm{R}-\mathrm{R}$ intervals; pNN50 $=$ the percentage of adjacent RR intervals with a difference of duration greater than $50 \mathrm{~ms}$; RMSSD = root-mean square of differences between adjacent normal RR intervals in a time interval. $\mathrm{LF}=$ low frequency; $\mathrm{HF}=$ high frequency; $\mathrm{LF} / \mathrm{HF}=$ low frequency/ high frequency ratio; $\mathrm{ms}=$ milliseconds; $\mathrm{nu}=$ normalized units; $\mathrm{ms}=$ millisecond.

$$
F(w) \propto w^{\alpha}
$$

To obtain sDFA, we calculate the spectral adaptation in exactly the same way as for spectral entropy using a Welch power spectrum with the same settings; but DFA rather than Shannon entropy is the algorithm applied.

\subsection{SMTM}

sMTM is founded on the elevated intensity of broadband noise in power spectra generated by irregular and chaotic signals. Multi-Taper Method (MTM) is useful for spectral estimation and signal reconstruction, of a timeseries of a spectrum that may contain broadband and line components. MTM is nonparametric since it does not apply an a priori, parameter dependent model of the process that generated the time-series under analysis. MTM reduces the variances of spectral estimates using a small set of tapers. Data are premultiplied by orthogonal tapers created to minimize the spectral leakage owing to the finite length of the time-series. A set of independent approximations of the power spectrum is calculated. Functions identified as slepian sequences, or discrete prolate spheroidal sequences (DPSS) [26] are a set of functions which optimize the tapers. They are defined as eigenvectors of a Rayleigh-Ritz minimization problem [27].

SMTM is the area between the MTM power spectrum and the baseline. We set the parameters for MTM at: sampling frequency of $1 \mathrm{~Hz}$, time bandwidth for the DPSS is set to 3, FFT length of 256 and, Thomson's adaptive 
nonlinear combination method to combine individual spectral estimates.

\subsection{Chaotic Forward Parameter}

The parameter [CFPx 1-7] is referred to as Chaotic Forward Parameter for the functions 1 to 7 below where it is applied to control and mental stimulation (through mathematical tasks) datasets. As sDFA responds to chaos in the opposite way to the others, we subtract its value from unity when applying here. All three chaotic global values have equal weighting. [CFP $x$ 1-7] are defined in the standard way as in Souza [13] and Vanderlei [18]

$$
\begin{aligned}
& 1\left[\mathrm{CFP}_{x}\right]=\left[\left(\left[\frac{\mathrm{sEntropy}}{\max (\text { sEntropy })}\right]\right)^{2}+\left(\left[\frac{\mathrm{sMTM}}{\max (\mathrm{sMTM})}\right]\right)^{2}\right. \\
& \left.+\left(1-\left[\frac{\mathrm{sDFA}}{\max (\mathrm{sDFA})}\right]\right)^{2}\right]^{\frac{1}{2}} \\
& 2\left[\mathrm{CFP}_{x}\right]=\left[\left(\left[\frac{\mathrm{sEntropy}}{\max (\text { sEntropy })}\right]\right)^{2}+\left(1-\left[\frac{\mathrm{sDFA}}{\max (\mathrm{sDFA})}\right]\right)^{2}\right]^{\frac{1}{2}} \\
& 3\left[\mathrm{CFP}_{x}\right]=\left[\left(\left[\frac{\text { sEntropy }}{\max (\text { sEntropy })}\right]\right)^{2}+\left(\left[\frac{\mathrm{sMTM}}{\max (\mathrm{sMTM})}\right]\right)^{2}\right]^{\frac{1}{2}} \\
& 4\left[\mathrm{CFP}_{x}\right]=\left[\left(\left[\frac{\mathrm{sMTM}}{\max (\mathrm{sMTM})}\right]\right)^{2}+\left(1-\left[\frac{\mathrm{sDFA}}{\max (\mathrm{sDFA})}\right]\right)^{2}\right]^{\frac{1}{2}} \\
& 5\left[\mathrm{CFP}_{x}\right]=\left[\left(1-\left[\frac{\mathrm{sDFA}}{\max (\mathrm{sDFA})}\right]\right)^{2}\right]^{\frac{1}{2}} \\
& 6\left[\mathrm{CFP}_{x}\right]=\left[\left(\left[\frac{\mathrm{sMTM}}{\max (\mathrm{sMTM})}\right]\right)^{2}\right]^{\frac{1}{2}} \\
& 7\left[\mathrm{CFP}_{x}\right]=\left[\left(\left[\frac{\text { sEntropy }}{\max (\text { sEntropy })}\right]\right)^{2}\right]^{\frac{1}{2}} \text {. }
\end{aligned}
$$

\section{RESULTS}

\subsection{Linear indices of HRV}

In Table 1, we observe that there was no significant change in the linear indices of HRV in the time and frequency domain between control and during the autonomic test.

\subsection{Statistical Analysis}

Before we analyse the data for significance, we must test the normal distribution of the data. Parametric statistics assume the data are normally distributed. Therefore, we use the mean as a measure of central tendancy. If we cannot normalize the data, we should not compare means. To test our assumptions of normality, we apply the Anderson-Darling [28] and Ryan-Joiner [29] tests. The Ryan-Joiner test is analogous to the Shapiro-Wilk [30,31] test. The Anderson-Darling test applies an empirical
FIQURE 1

\section{Control ( $\mathbf{N}=\mathbf{2 4})$}

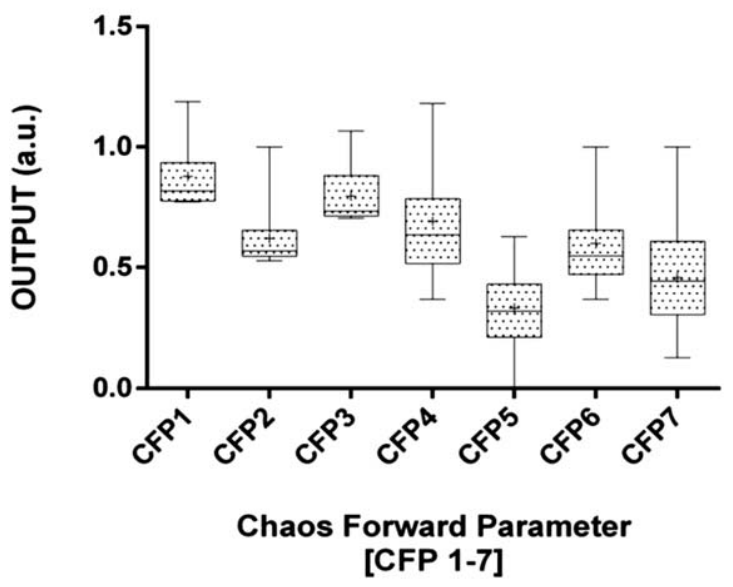

The boxplot illustrates the mean values and standard deviation of CFP for control subjects ECG RR intervals. The mean value is indicated by the $(+)$ symbol in the boxplot. There are 256 RR intervals across 24 subjects.

cumulative distribution function, whereas the Ryan-Joiner test is a correlation-based test. The tests indicate mainly non-normal distribution so we apply the Kruskal-Wallis [32] test of significance. It is referred to as nonparametric. The most significant combinations of "chaotic globals" are all except CFP 2 and CFP 3. From control to mathematical

\section{FIGURE 2}

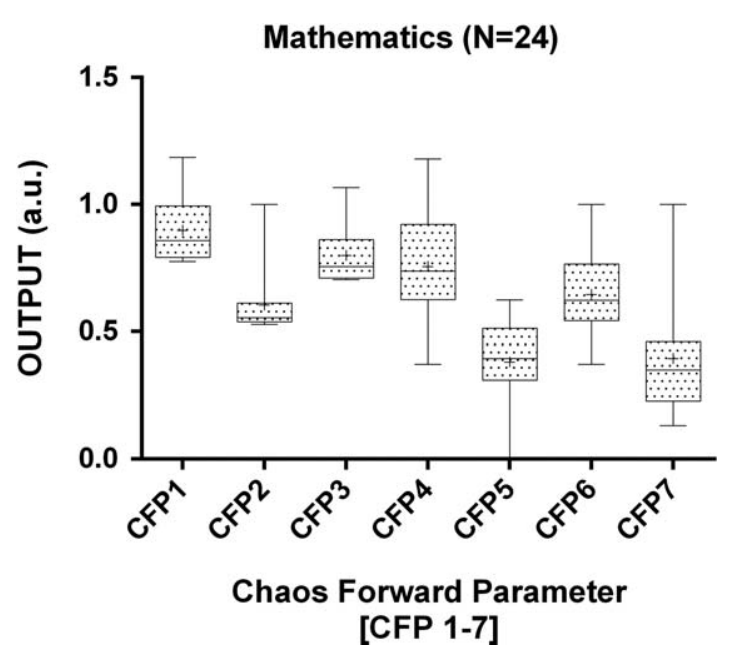

The boxplot illustrates the mean values and standard deviation of CFP for the ECGs RR intervals of subjects with mental stimulation via mathematical tests. Mean values are indicated by the $(+)$ symbol. All 24 subjects have data of length 256 RR intervals. 
mental stimulation, the values for CFP 2 and CFP 7; both decrease in chaotic response (Figure 1). Therefore, they can be excluded. CFP 3 increases but it is not statistically significant. CFP 2 is also not significant by $P$-value.

CFP 1 and CFP 4 to 6; all increase in chaosity as expected (Figure 2). They are statistically significant by nonparametric techniques. For these four combinations, we have significnace at the level $P<0.2$ for all. For the principal component analysis (PCA; Section 3.3), we need to assess these combinations of CFP. CFP 4 to 6 all perform strongly on the first principal componenet (PC1) but CFP 1 outperforms these on the second component (PC2). That aside we recommend that CFP 1 is the most influencial overall. We do not need to assess the third component (PC3) or higher as the scree plot is steep.

\subsection{Principal Component Analysis}

PCA [33]; a multivariate statistical technique can be applied here (See Tables 2 and 3). We have the values of [CFP] for seven groups for 24 subjects who are undergoing mental stimulation by mathematical tasks; hence a grid of 7 by 24 to be assessed. The PC1 has a variance (eigenvalue) of 4.4503 and accounts for $63.6 \%$ of the total variance. The PC2 has an eigenvalue of 2.5425 accounting for $99.9 \%$ of total variance. The second component has a proportion of influence of $36.3 \%$. Therefore, we can assume that most variance is acheived in the first two components.

When we observe the results of PCA; and recalling the Kruskal-Wallis statistical analysis we consider all combinations except CFP 2 and CFP 3. CFP 7 is excluded on the basis that the chaosity decreases from control to experimental, which is not to be expected. CFP 4 to 6 all perform well on the first component, but CFP 1 performs best on the second component. Since most influence is based on the first component (63.6\%) then we can assume (excluding CFP 7 as discussed); generally that the best combinations are those with single spectral parameters sDFA and sMTM alone. However, previous stuudies indicate that CFP 1 is the most significant overall. The best balance is acheived in both Kruskal-Wallis and multivariate analysis for CFP 1.

\section{DISCUSSION}

The mathematical analysis is considered such that it can be applied appropriately, not only retrospectively but also in a laboratory and clinical setting-online in realtime situations. We undertake the analysis for control and subjects undergoing mental stimulation. The algorithms compute significance at the level $P<0.2$ for all combinations except 2 and 3. CFP 2 and 7 are rejected due to decreasing chaotic response. PCA indicates that CFP 4 to 6 are most influencial for the PC1 while CFP 1 is much

\section{TABLE 2}

The table below shows the mean values and standard deviation of the Chaos Forward Parameters [1-7] for the normal and subjects undergoing mathematical mental tasks (referred to as "Math") subjects RR intervals

\begin{tabular}{lccccc}
\hline & $\begin{array}{c}\text { Mean } \\
\text { Control } \\
(n=24)\end{array}$ & $\begin{array}{c}\text { Standard } \\
\text { Deviation } \\
\text { Control }\end{array}$ & $\begin{array}{c}\text { Mean } \\
\text { Math } \\
(n=24)\end{array}$ & $\begin{array}{c}\text { Standard } \\
\text { Deviation } \\
\text { Math }\end{array}$ & $\begin{array}{c}\text { Kruskal-Wallis } \\
(P \text {-value })\end{array}$ \\
\hline CFP] 1 & 0.8776 & 0.1364 & 0.8985 & 0.1218 & 0.2085 \\
CFP 2 & 0.6201 & 0.1175 & 0.6038 & 0.1171 & 0.4394 \\
CFP 3 & 0.7975 & 0.1165 & 0.7988 & 0.1072 & 0.7571 \\
CFP 4 & 0.6914 & 0.2310 & 0.7555 & 0.2175 & 0.1609 \\
CFP 5 & 0.3345 & 0.1683 & 0.3825 & 0.1649 & 0.1975 \\
CFP 6 & 0.5996 & 0.1787 & 0.6461 & 0.1658 & 0.1518 \\
CFP 7 & 0.4579 & 0.2264 & 0.3961 & 0.2249 & 0.2047 \\
\hline
\end{tabular}

The number of RR intervals is 256. Kruskal-Wallis test of significance is applied to results giving a $P$-value.

more influential than the others for the PC2. Only the first two components are relevant due to the steep scree plot. Regarding the classical indices, there were no significant differences for any HRV parameters during metal stress.

Mental task load was shown to induce an autonomic reaction. Previous studies have reported how mental task load changes HRV properties, because experimental evidence of an association between autonomic nervous activity and cardiac regulation has been suggested [18]. Heart rate commonly enhances during performance of mental tension. Conversely, it reduces during fatigue [18]. Based on previous studies that focused on HRV indices in the time and frequency domain, mental task load reduced the power of both the low frequency (LF) and high frequency (HF) [22]. In this context, the LF (0.04-0.15 Hz) component of HRV is indicated to represent both sympathetic and parasympathetic cardiac modulation, with a predominance of the sympathetic component. The HF $(0.2-0.5 \mathrm{~Hz})$ component of HRV is thought to reflect parasympathetic nerve tone [18].

In these circumstances, we expected reduced HRV during performance of the seleced mental task. Although some indices almost reached statistical significance, tending to a reduced parasympathetic modulation of the heart during the mental stress, we failed to report significant responses of the global chaotic parameters of HRV to the mental arithmetic overload test. Several studies only emphases on mental or physical load, however, only a few consider both. In this context, HRV has been widely used to investigate the reaction to mental demands on the ANS [18]. In our study, HRV was compared between before and during performance of a specific mental task because HRV is sensitive to alterations in mental state [34]. Mental 


\section{TABLE 3}

The table below is the Principal Component Analysis for CFP for seven groups for 24 subjects who are subjected to mental stimulation through mathematical tasks

\begin{tabular}{|c|c|c|c|c|c|c|c|}
\hline Variable & PC1 & PC2 & PC3 & PC4 & PC5 & PC6 & PC7 \\
\hline CFP1 & 0.198 & -0.570 & 0.335 & -0.604 & 0.273 & 0.205 & -0.208 \\
\hline CFP2 & -0.302 & -0.483 & 0.511 & 0.546 & -0.012 & 0.077 & 0.332 \\
\hline CFP3 & -0.019 & -0.626 & -0.552 & 0.177 & -0.112 & -0.429 & -0.274 \\
\hline CFP4 & 0.467 & -0.108 & 0.036 & -0.242 & -0.367 & -0.345 & 0.676 \\
\hline CFP5 & 0.473 & 0.031 & 0.338 & 0.262 & -0.567 & 0.081 & -0.513 \\
\hline CFP6 & 0.460 & -0.148 & -0.411 & 0.289 & 0.166 & 0.664 & 0.213 \\
\hline CFP7 & -0.464 & -0.126 & -0.193 & -0.309 & -0.655 & 0.449 & 0.074 \\
\hline
\end{tabular}

PC1 represents the first principal component, PC2 the second; until the seventh component PC7.

stress is expected to reduce HF power of heartbeat interval time-series and other indices that correspond to the parasympathetic modulation of the heart, such as pNN50 and RMSSD, as already previously mentioned [35]. Conversely, we observed no significant changes in those indices between before, during and after the mental task used in our study. We may conclude that the single mental arithmetic overload test does not cause enough stimulation on HRV; since a previous study indicated that the addition of mental tasks to a physical computer task does not cause additional effect on HRV parameters related to autonomic modulation-showing that the physical demands have a major influence on HRV responses while the effect of the mental task is not significant [36].

This study has limitations; spectral entropy and sDFA, lose phase information. It may be possible to apply these spectral values in combination with shannon entropy, DFA, correlation dimension [37-39], approximate entropy [40-43], Higuchi fractal dimension [44], and sample entropy [45-47]. Indeed with the exception of the Higuchi fractal dimension (which is not compatible with power spectra) these could all be applied as additional spectral derivatives.

For spectral entropy and SDFA, the Welch power spectrum could be replaced by the more sensitive multi-taper spectrum. The DPSS of the MTM of sMTM could be optimized, and the chaotic global technique parameters could be weighted as here they are equally weight at unity.

\section{CONCLUSION}

The results discussed indicate that in these extremely short time-series the chaotic global techniques are unable to distinguish between the two data types-control and mental stimulation. However, it is concluded that the chaotic global combination CFP 1 with all three spectral parameters is the more sensitive to detect changes compared to the seven combinations but only achieves the level $P$-value up to 0.2 . It is recommended that the timeseries be increased to $1000 \mathrm{RR}$ intervals (at least $20 \mathrm{~min}$ of recordings) and standard nonlinear methods be introduced in combination with spectral factors as a way of increasing the statistical significance by $P$-value.

\section{ACKNOWLEDGMENTS}

The authors declare that there is no conflict of interests regarding the publication of this article. The study received financial support from FAPESP (number 2013/ 05102-6).

\section{REFERENCES}

1. Seely, A.J.; Macklem, P. Fractal variability: An emergent property of complex dissipative systems. Chaos 2012, 22, 013108.

2. Ho, M.-W. The rainbow and the worm: The physics of organisms; Singapore: World Scientific, 2008.

3. Garner, D.M.; Ling, B.W.K. Measuring and locating zones of chaos and irregularity. J Syst Sci Complex 2014, 27, 494-506.

4. Mackey, M.C.; Milton, J.G. Dynamical diseases. Ann N Y Acad Sci 1987, 504, 16-32.

5. Skinner, J.E.; Pratt, C.M.; Vybiral, T. A reduction in the correlation dimension of heartbeat intervals precedes imminent ventricular fibrillation in human subjects. Am Heart J 1993, 125, 731-743.

6. Burykin, A.; Costa, MD.; Peng, C.K.; Goldberger, A.L.; Buchman, T.G. Generating signals with multiscale time irreversibility: The asymmetric weierstrass function. Complexity 2011, 16, 29-38.

7. Peng, C.K.; Havlin, S.; Hausdorff, J.M.; Mietus, J.E.; Stanley, H.E.; Goldberger, A.L. Fractal mechanisms and heart rate dynamics. Long-range correlations and their breakdown with disease. J Electrocardiol 1995, 28, 59-65. 
8. Stead, M.; Worrell, G.A.; Litt, B. Frequency and dependence of long range temporal correlations in human hippocampal energy fluctuations. Complexity 2005, 10, 35-44.

9. Zyczkowski, K. Renyi extrapolation of Shannon entropy. Open Syst Inf Dyn 2003, 3, 297-310.

10. Shannon, C.E. A mathematical theory of communication. ACM SIGMOBILE Mobile Comput Commun Rev 2001, 5, 3-55.

11. Ryabko, B.; Reznikova, Z. Using Shannon entropy and Kolmogorov complexity to study the communicative system and cognitive capacities in ants. Complexity 1996, 2, 37-42.

12. Johnson, R.; Shore, J. Which is the better entropy expression for speech processing:-S log S or log S? IEEE Trans Acoust Speech Signal Process 1984, 32, 129-137.

13. Souza, N.M.; Vanderlei, L.C.; Garner, D.M. Risk evaluation of diabetes mellitus by relation of chaotic globals to HRV. Complexity 2015, 20, 84-92.

14. Alvarez, D.; Hornero, R.; Marcos, J.; Del Campo, F.; Lopez, M. Spectral analysis of electroencephalogram and oximetric signals in obstructive sleep apnea diagnosis. Conf Proc IEEE Eng Med Biol Soc 2009, 2009, 400-403.

15. Morgaz, J.; Granados, M.D.; Dominguez, J.M.; Navarrete, R.; Fernandez, A.; Galan, A.; Munoz, P.; Gomez-Villamandos, R.J. Evaluation of spectral entropy to measure anaesthetic depth and antinociception in sevoflurane-anaesthetised Beagle dogs. Vet J 2011, 188, 352-355.

16. Ziegler, D. Diabetic cardiovascular autonomic neuropathy: Prognosis, diagnosis and treatment. Diabetes Metab Rev 1994, 10, 339-383.

17. Vinik, A.I.; Ziegler, D. Diabetic cardiovascular autonomic neuropathy. Circulation 2007, 115, 387-397.

18. Vanderlei, F.M.; Vanderlei, L.C.; Garner, D.M. Chaotic global parameters correlation with heart rate variability in obese children. J Hum Growth Dev 2014, 24, 24-30.

19. Alkan, A.; Kiymik, M.K. Comparison of AR and Welch methods in epileptic seizure detection. J Med Sys 2006,6 , 413-419.

20. Ghil, M. The SSA-MTM Toolkit: Applications to analysis and prediction of time series. Appl Soft Comput 1997, 3165, 216230.

21. Percival, D.B.; Walden, A.T. Spectral Analysis for Physical Applications: Multitaper and Conventional Univariate Techniques; Cambridge University Press, Cambridge, 1993.

22. Hocker, J.; Raitschew, B.; Meybohm, P.; Broch, O.; Stapelfeldt, C.; Gruenewald, M.; Cavus, E.; Steinfath, M.; Bein, B. Differences between bispectral index and spectral entropy during xenon anaesthesia: A comparison with propofol anaesthesia. Anaesthesia 2010, 65, 595-600.

23. Stilp, C.E.; Kiefte, M.; Alexander, J.M.; Kluender, K.R. Cochlea-scaled spectral entropy predicts rate-invariant intelligibility of temporally distorted sentences. J Acoust Soc Am 2010, 128, 2112-2126.

24. Peng, C.K.; Havlin, S.; Stanley, H.E.; Goldberger, A.L. Quantification of scaling exponents and crossover phenomena in nonstationary heartbeat time series. Chaos 1995, 5, 82-87.

25. Donaldson, G.C.; Seemungal, T.A.; Hurst, J.R.; Wedzicha, J.A. Detrended fluctuation analysis of peak expiratory flow and exacerbation frequency in COPD. Eur Respir J 2012, 40, 1123-1129.

26. Slepian, S. Prolate spheroidal wave functions, Fourier analysis and uncertainty, V, The discrete case. Bell Syst Tech J 1978, 57, 1371-1430.

27. Gould, S.H. Variational Methods for Eigenvalue Problems: An Introduction to the Methods of Rayleigh, Ritz, Weinstein, and Aronszajn; Courier Dover Publications, Mineola, NY, 1995.

28. Anderson, T.W.; Darling, D.A. A test of goodness of fit. J Am Stat Assoc 1954, 49, 765-769.

29. Ryan, T.A.; Joiner, B.L. Normal probability plots and tests for normality. Minitab Statistical Software: Technical Reports. The Pennsylvania State University, State College, PA. Available from MINITAB, 1976.

30. Razali, N.M.; Wah, Y.B. Power comparisons of shapiro-wilk, kolmogorov-smirnov, lilliefors and anderson-darling tests. J Stat Model Anal 2011, 2, 21-33.

31. Royston, P. Approximating the Shapiro-Wilk W-Test for non-normality. Stat Comput 1992, 2, 117-119.

32. Kruskal, W.H.; Wallis, W.A. Use of ranks in one-criterion variance analysis. J Am Stat Assoc 1952, 260, 583-621.

33. Jolliffe, I.T. Principal Component Analysis, Springer Series in Statistics; Springer, Berlin Heidelberg, 2002.

34. Abreu, L.C. Heart rate variability as a functional marker of development. J Hum Growth Dev 2012, 22, $279-281$.

35. Zhong, X.; Hilton, H.J.; Gates, G.J.; Jelic, S.; Stern, Y.; Bartels, Y. Increased sympathetic and decreased parasympathetic cardiovascular modulation in normal humans with acute sleep deprivation. J Appl Physiol 2005, 98, 2024-2032.

36. Fauvel, J.P.; Cerutti, C.; Quelin, P.; Laville, M.; Gustin, M.P.; Paultre, C.Z.; Ducher, M. Mental stress- induced increase in blood pressure is not related to barore flex sensitivity in middle-aged healthy men. Hypertension 2000, 35, 887-891.

37. Van Leeuwen, P., Bettermann, H., An der, Heiden U., Kummell, H.C. Circadian aspects of apparent correlation dimension in human heart rate dynamics. Am J Physiol 1995, 269, H130-H134.

38. Skinner, J.E.; Carpeggiani, C.; Landisman, C.E.; Fulton, K.W. Correlation dimension of heartbeat intervals is reduced in conscious pigs by myocardial ischemia. Circ Res 1991, 68, 966-976.

39. Burioka, N.; Cornelissen, G.; Halberg, F.; Kaplan, D.T. Relationship between correlation dimension and indices of linear analysis in both respiratory movement and electroencephalogram. Clin Neurophysiol 2001, 112, 1147-1153.

40. Pincus, S. Approximate entropy (ApEn) as a complexity measure. Chaos 1995, 5, 110-117.

41. Hu, X.Y.; Wan, L.; Xie, D.Y. Effects of noise on the approximate entropy of fractional Brownian motion sequence. Appl Mech Mater 2014, 444, 698-702.

42. Ocak, H. Automatic detection of epileptic seizures in EEG using discrete wavelet transform and approximate entropy. Expert Syst Appl 2009, 36, 2027-2036. 
43. Srinivasan, V.; Eswaran, C.; Sriraam, N. Approximate entropy-based epileptic EEG detection using artificial neural networks. IEEE Trans Inf Technol Biomed 2007, 11, 288-295.

44. Anier, A.; Lipping, T.; Melto, S.; Hovilehto, S. Higuchi fractal dimension and spectral entropy as measures of depth of sedation in intensive care unit. Conf Proc IEEE Eng Med Biol Soc 2004, 1, 526-529.

45. Richman, J.S.; Lake, D.E.; Moorman, J.R. Sample entropy. Methods Enzymol 2004, 172-184.

46. Lake, D.E.; Richman, J.S.; Griffin, M.P.; Moorman, J.R. Sample entropy analysis of neonatal heart rate variability. Am J Physiol Regul Integr Comp Physiol 2002, 283, R789-R797.

47. Chen, Y.; Pham, T.D. Sample entropy and regularity dimension in complexity analysis of cortical surface structure in early Alzheimer's disease and aging. J Neurosci Methods 2013, 215, 210-217. 